\title{
Low-frequency noise in single electron tunneling transistor
}

Tavkhelidze, A.N.; Mygind, Jesper

Published in:

Journal of Applied Physics

Link to article, DOI:

10.1063/1.366685

Publication date:

1998

Document Version

Publisher's PDF, also known as Version of record

Link back to DTU Orbit

Citation (APA):

Tavkhelidze, A. N., \& Mygind, J. (1998). Low-frequency noise in single electron tunneling transistor. Journal of Applied Physics, 83(1), 310-317. https://doi.org/10.1063/1.366685

\section{General rights}

Copyright and moral rights for the publications made accessible in the public portal are retained by the authors and/or other copyright owners and it is a condition of accessing publications that users recognise and abide by the legal requirements associated with these rights.

- Users may download and print one copy of any publication from the public portal for the purpose of private study or research.

- You may not further distribute the material or use it for any profit-making activity or commercial gain

- You may freely distribute the URL identifying the publication in the public portal

If you believe that this document breaches copyright please contact us providing details, and we will remove access to the work immediately and investigate your claim. 


\title{
Low-frequency noise in single electron tunneling transistor
}

\author{
A. N. Tavkhelidze ${ }^{\text {a) }}$ and J. Mygind ${ }^{\text {b) }}$ \\ Department of Physics, Technical University of Denmark, DK-2800 Lyngby, Denmark
}

(Received 7 April 1997; accepted for publication 30 September 1997)

\begin{abstract}
The noise in current biased aluminium single electron tunneling (SET) transistors has been investigated in the frequency range of $5 \mathrm{mHz}<f<30 \mathrm{~Hz}$. A refined high frequency (HF) shielding including resistive coaxial lines, that prevents spurious electromagnetic radiation and especially high energy photons emitted by the $4.2 \mathrm{~K}$ environment from reaching the sample, allows us to study a given background charge configuration for many hours below $\approx 100 \mathrm{mK}$. The noise at relatively high frequencies originates from internal (presumably thermal equilibrium) charge fluctuations. For $f \geqslant 10 \mathrm{~Hz}$, we find the same input charge noise, typically $Q_{N}=5 \times 10^{-4} \mathrm{e} / \mathrm{Hz}^{1 / 2}$ at $10 \mathrm{~Hz}$, with and without the HF shielding. At lower frequencies, the noise is due to charge trapping, and the voltage noise pattern superimposed on the $V\left(V_{g}\right)$ curve (voltage across transistor versus gate voltage) strongly depends on the background charge configuration resulting from the cooling sequence and eventual radio frequency (rf) irradiation. The measured noise spectra which show both $1 / f$ and $1 / f^{1 / 2}$ dependencies and saturation for $f<100 \mathrm{mHz}$ can be fitted by two-level fluctuators with DebyeLorentzian spectra and relaxation times of order seconds. In some cases, the positive and negative slopes of the $V\left(V_{g}\right)$ curve have different overlaid noise patterns. For fixed bias on both slopes, we measure the same noise spectrum, and believe that the asymmetric noise is due to dynamic charge trapping near or inside one of the junctions induced when ramping the junction voltage. Dynamic trapping may limit the high frequency applications of the SET transistor. Also reported on are the effects of rf irradiation and the dependence of the SET transistor noise on bias voltage. () 1998 American Institute of Physics. [S0021-8979(98)06301-4]
\end{abstract}

\section{INTRODUCTION}

Single electron tunneling (SET) devices are considered as potential candidates for future high density electronics because of their unique properties such as very low power operation and subelectron charge sensitivity (see Ref. 1 for review). Their long term stability and general noise properties when integrated in real circuits are of crucial importance in most SET applications such as electrometers, charge amplifiers, electron traps, and current standards (see Refs. $2-5$ and references therein). The basic three-terminal component is the SET transistor which consists of two nearly identical series connected ultrasmall high-resistance tunnel junctions with capacitance $C_{1} \approx C_{2} \ll e^{2} / k_{B} T$ and resistance $R_{1} \approx R_{2}$ $\gg R_{Q}=h / e^{2}$. The charge of the interconnecting metallic island can be regulated continuously by applying a voltage $V_{g}$ to a gate electrode which is capacitively coupled to the island by a small gate capacitance $C_{g}<C_{1}, C_{2}$. If the total capacitance $C_{\Sigma}=C_{1}+C_{2}+C_{g}$ of the island is so small that admission of one electron, giving the additional charging energy $E_{C}=e^{2} / 2 C_{\Sigma}$, changes its electrostatic energy significantly, the system shows so-called Coulomb blockade. With a dc bias voltage $V$ applied across the transistor the quantized tunnel current of single electrons becomes $e$ periodic with the period $C_{g} V_{g}=e$, and in the ideal case $(T=0$, orthodox theory Ref. 1), the current may be reduced to zero for $V$ $\leqslant V_{C}=e / C_{\Sigma}$; the Coulomb blockade threshold. In real SET,

\footnotetext{
${ }^{a}$ Permanent address: Department of Physics, Tbilisi State University, Chavchavadze Ave. 2, 380097 Tbilisi, Georgia.

b)Electronic mail: myg@mips.fys.dtu.dk
}

transistors both noise and cotunneling ${ }^{6}$ prevent full blockade, and the bias condition determines which of the two junctions mainly limits the tunnel current.

Most metal SET devices are $\mathrm{Al} / \mathrm{Al}_{2} \mathrm{O}_{3} / \mathrm{Al}$ tunnel junctions fabricated by shadow evaporation with masks made by electron beam lithography. This technique only allows for production of junctions with capacitance down to $\approx 10 \mathrm{aF}$ implying that operational temperatures need to be in the millikelvin range. Other materials, ${ }^{7,8}$ fabrication techniques (see Ref. 2 for review also on semiconductor based junctions), and structures ${ }^{9}$ are being developed. Recently junctions with capacitance as low as $C \approx 0.1 \mathrm{aF}$, showing Coulomb blockade and Coulomb staircase at room temperature, have been fabricated by nano-oxidation of a Ti film using the tip of a scanning tunneling microscope (STM) ${ }^{10}$

The major obstacle to future applications of the SET transistor is fluctuations in the excess charge on the island leading to a high level of $1 / f^{\alpha}$ type noise which at low frequencies dominates other intrinsic noise sources such as shot noise and resistance fluctuations. Current or voltage noise with $1 / f^{\alpha}$ ( $\alpha$ between 0.5-1.5) frequency dependent noise has been observed in almost all electronic devices, each having a characteristic frequency range and spectral density. According to Hooge's empirical law, ${ }^{11}$ the spectral density varies inversely with the total number of carriers in the system. Except for a few systems, the physical origin of the $1 / f^{\alpha}$ noise is not understood and in most cases it is not even clear whether it is a volume or a surface effect. Theoretical models based on surface trapping and equilibrium thermal energy exchange with the environment have been extensively discussed. For the SET transistors, the random pulse train model is of particular interest due to the experimental obser- 
vations of two-level fluctuations of the island charge,${ }^{3}$ conveniently described by the two level fluctuator [TLF, see below]. Usually the charge noise referred to the input $Q_{N}$ is given in units of e/ $\mathrm{Hz}^{1 / 2}$. Alternatively one may use the energy resolution of the SET transistor $E_{N}=Q_{N}^{2} / 2 C_{\Sigma}$. The terminology is analogous to flux noise contra energy resolution of (SQUIDs), but may lead to confusion when comparing slopes of noise spectra.

Generally, the charge fluctuations are believed to be caused by internal instabilities associated with random shifts of the intrinsic background charges near the island or inside the tunnel junctions (see Chaps. 3, 7, and 9 in Ref. 2). Several attempts have been made to understand the nature ${ }^{12}$ of the background charge noise and to eliminate it by using various substrates and different electrode materials, ${ }^{13}$ see also the last paper in Ref. 6. A natural physical explanation is stochastic occupation of charge traps analogous to the mechanism leading to the $1 / f^{1 / 2}$ voltage noise pertinent to metal-oxide-semiconductor field-effect transistor (MOSFETs). ${ }^{11,14,15}$

Alternatively, in SET devices, the $1 / f^{\alpha}$ noise may be due to externally induced charge fluctuations caused by spurious environmental electrical noise and electromagnetic radiation, and in particular, photon assisted tunneling and/or charge detrapping induced by the thermal radiation emitted from the $T_{e}=4.2 \mathrm{~K}$ parts of the dilution refrigerator. ${ }^{16}$ This radiation is in the frequency range up to $k_{B} T_{e} / h \approx 90 \mathrm{GHz}$, where $k_{B}$ is Boltzmann's constant, and $h$ is Planck's constant. The energy $E=\mathrm{hf}$ of $90 \mathrm{GHz}$ photons is comparable to the charging energy $E_{C}=e^{2} / 2 C_{\Sigma}$ of junctions with typical capacitance $C_{\Sigma} \approx 200 \mathrm{aF}$, and is $\approx 100$ times larger than the energy of the photons (with frequencies from dc to $k_{B} T_{s} / h$ ) emitted by the sample itself being in thermal equilibrium at say $T_{s}$ $\approx 40 \mathrm{mK}$.

As described below, the very careful electrical and thermal shielding of our setup permits us to "freeze" a given background charge noise pattern when cooling to temperatures below a few $100 \mathrm{mK}$. This enables us to study for many hours different background charge or charge trap configurations resulting from a particular cooling sequence or repetitive rf irradiation. We find that charging effects induced by these nonequilibrium photons clearly influence the noise spectrum at frequencies below a few $\mathrm{Hz}$, but we are unable to tell whether detrapping of the charge carriers, or photon assisted tunneling of electrons across the current limiting junction (eventually helped by traps inside the junction barrier) is the dominant mechanism for creating a new background charge noise pattern.

\section{JUNCTIONS AND DC CHARACTERIZATION}

The noise measurements were done on $100 \times 100 \mathrm{~nm}^{2}$ $\mathrm{Al} / \mathrm{Al}_{2} \mathrm{O}_{3} / \mathrm{Al}$ tunnel junctions $\left(C_{1} \approx C_{2} \approx 350 \mathrm{aF}, R_{1} \approx R_{2}\right.$ $\approx 60 \mathrm{k} \Omega$ ) fabricated in a single vacuum cycle by conventional shadow evaporation on an nonoxidized doped silicon wafer using masks made by electron beam lithography. Each $9 \times 9 \mathrm{~mm}^{2}$ chip contains eight different SET transistor configurations. In order to reduce the intrinsic surface charge traps, almost all of the chip area is covered with aluminium (serving also as dc contacts). Only close to the SET structures open $\mathrm{Si}$ areas remain. The conductivity of the Si substrate protects the transistors electrically at room temperature. Most samples have sustained several cryogenic thermal cycles, remounts and storage for over a year with nearly unchanged parameters in an ordinary water-free exsiccator at ambient temperature.

In the experiments, the SET transistor is symmetrically current biased. The transistor then operates as a voltage to voltage converter where the voltage gain $K_{v}=d V / d V_{g}$ is the slope of the $V\left(V_{g}\right)$ modulation curve. The sensitivity to $V_{g}$ depends on the bias current $I_{B}$ and is largest slightly below the Coulomb blockade voltage $V_{C}=e / C_{\Sigma}$. In order not to confuse the presentation with different junction properties, we in the following only give results obtained on a particular SET transistor with typical parameters and characteristics. We have chosen a fairly large junction with relatively low charging energy in order to make it more sensitive to external radiation. An accurate determination of the offset voltage was made by calculating $V_{C}(V)=V-I_{B}(d V / d I)^{17}$ at different bias points in the current-voltage $(I-V)$ curve. We find $V_{C}(V)$ to be nearly constant for voltages above $2 \mathrm{mV}$ yielding the value $V_{C}=220 \mu \mathrm{V}$ and $C_{\Sigma}=730 \mathrm{aF}$. The $C_{g}$ and $C_{1,2}$ can be found ${ }^{18}$ from the period $\Delta V_{g}$ and the slopes $d V / d V_{g}$ of the $V\left(V_{g}\right)$ curve using $C_{g}=\left(e / \Delta V_{g}\right)$ and $C_{1,2}$ $=\left(e / \Delta V_{g}\right)_{p, n} d V_{g} / d V$, where the $p$ and $n$ denote positive and negative slope. We find $C_{1}=350, C_{2}=380$, and $C_{g}$ $=15 \mathrm{aF}$, i.e., $C_{\Sigma}=750 \mathrm{aF}$ in good agreement with the value found by the first method. From the slope of the $I-V$ curve, we get the asymptotic normal-state resistance $R_{\Sigma}=120 \mathrm{k} \Omega$ giving $R_{1} \approx R_{2} \approx 60 \mathrm{k} \Omega$.

\section{EXPERIMENTAL SETUP}

A balanced electronic system is used to symmetrically current bias the SET transistor. The current source consists of a regulated voltage source with two antipolar symmetrical outputs, and two high-resistance resistors $R_{b}$ connected in series with the sample. Low noise instrumentation amplifiers (voltage gain 100, cut-off frequency at $30 \mathrm{~Hz}$ ) with ultrahighresistance differential inputs are used to simultaneously measure the voltage $V$ across the sample and the output voltage $V_{s}$ of the source. The current through the sample is $I_{B}$ $=\left(V_{s}-V\right) / 2 R_{b}$. The gate voltage $V_{g}$ is measured by a third instrumentation amplifier. The critical amplifiers, filters, and a matrix switch for connection to the various sample terminals are enclosed in a small metal box on top of the KelvinOx ${ }^{\circledR}$ dilution refrigerator. The three outputs from the box $\left(V, V_{g}\right.$, and $\left.I_{B}\right)$ are fed to a 40 channel multiplexer followed by a commercial low noise amplifier (with adjustable lowpass filter) and finally measured by a digital voltmeter (integration time typically $200 \mathrm{~ms}$ ). The multiplexer also handles the signals from other transducers in the setup (thermometers, pressures, applied magnetic field, etc.). The monitoring of the thermometers placed in the refrigerator allows us to follow and regulate the cooling sequence of the sample. A commercial computer system (LabView $\left.{ }^{\circledR}\right)$ with IEEE interface with an optical link to a standard PC is used for instrument control and data collection. The source and gate voltages are supplied from mercury batteries or from balanced dc 
sources regulated by the IEEE interface. Built-in rechargeable batteries serve as a power supply for the instrumentation amplifiers.

Inside the cryostat, all wires from the room temperature electronics to the sample (and thermometers) are resistive twisted pairs thermally anchored at $4.2,1.2$, and $0.6 \mathrm{~K}$ to the metallic mixing chamber. The sample holder mounted onto the mixing chamber is specially designed to exclude the high frequency components of the $4.2 \mathrm{~K}$ thermal radiation. The only electrical connections from the mixing chamber connector to the chip are twenty $25-\mathrm{cm}$-long resistive stainless steel Thermocoax ${ }^{\circledR}$ cables with $>100 \mathrm{~dB}$ power attenuation above $20 \mathrm{GHz} .{ }^{19}$ A matrix system of twenty 25 -mm-long and 0.2 -mm-diam buckling wires each soldered to the $0.2-\mathrm{mm}$ diam center conductor of the coax cables establishes the connections to the contacts on the chip. Both sample and cables are enclosed in a thick copper can mounted on the mixing chamber. The can serves both as thermal and electrical shield and secures that the whole cable length is at the same temperature as the sample. Two calibrated resistance thermometers mounted on the mixing chamber are measured by a compensating rf bridge, and a standard temperature stabilizer can be used to maintain a given temperature. The entire dilution refrigerator is enclosed in a stainless steel vacuum can immersed in a liquid ${ }^{4} \mathrm{He}$ bath metal cryostat. During the noise measurements, a persistent mode superconducting magnet is used to apply a magnetic field perpendicular to the chip to suppress superconductivity of the sample.

\section{NOISE MEASUREMENTS; RESULTS AND DISCUSSION}

The $V\left(V_{g}\right)$ curve is measured for fixed $I_{B}$ by linearly ramping up the gate voltage usually from approx. -10 to $+10 \mathrm{mV}$ in 500 equidistant steps. Typically it takes approx. $10 \mathrm{~min}$ to record one curve. The $V\left(V_{g}\right)$ curve appears as a periodic (nearly sinusoidal) smooth modulation curve [see Fig. 1(a)] overlaid with a voltage noise $V_{N}(t)$. Depending on $I_{B}$, the modulation curve has a dc voltage offset of $\approx 1 \mathrm{mV}$. As discussed below, the sensitivity to charge noise is proportional to the voltage gain $K_{V}$ and usually noise measurements are done for fixed $V_{g}$ near the maximum slope of the $V\left(V_{g}\right)$ curve. $V_{N}(t)$ is measured by a digital voltmeter and/or by a spectrum analyzer (HP 3561A) and both the PC based data collection system (LabView $®)$ and the spectrum analyzer calculate the noise spectral density $V_{N}(f)$ using standard fast fourier transform (FFT) algorithms.

The fact that the voltage noise measured at a fixed frequency strictly follows the slope of the $V\left(V_{g}\right)$ curve proves that the SET transistor noise is dominated by fluctuations in the charge on (or near) the metallic island between the two junctions. With this assumption and when the noise amplitude is small compared to the $V\left(V_{g}\right)$ modulation, the voltage noise $V_{N}$ can be converted to input charge noise $Q_{N}$ using

$$
Q_{N}=C_{g}\left(K_{V}\right)^{-1} V_{N} \text {. }
$$

Figure 2 shows $Q_{N}$ measured $10 \mathrm{~Hz}$ and the corresponding conversion factor $K_{V}$ versus the normalized bias voltage $V /\left(e / C_{\Sigma}\right)$. Actually we recorded the $V\left(V_{g}\right)$ curve for a fixed bias current, adjusted the gate voltage to maximum $K_{V}$ on
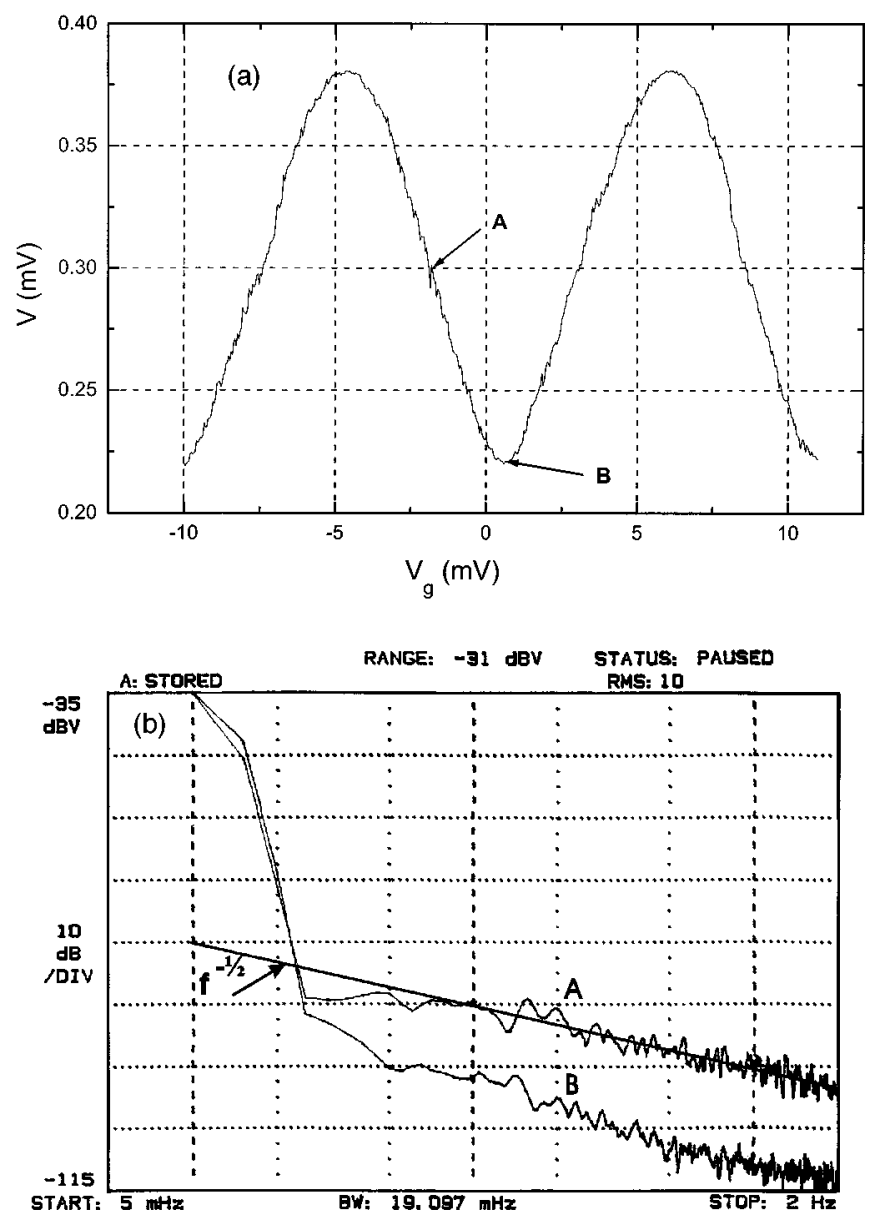

FIG. 1. (a) Typical low-noise $V\left(V_{g}\right)$ curve recorded over 9 min with $V_{g}$ incremented from -10 to $+12 \mathrm{mV}\left(I_{B}=0.2 \mathrm{nA}, T=70 \mathrm{mK}, B=0.5 \mathrm{~T}\right)$ and (b) the corresponding voltage noise spectra $(5 \mathrm{mHz} \leqslant f \leqslant 2 \mathrm{~Hz}$, numerical average of 10 spectra each recorded during $200 \mathrm{~s})$ measured with the SET transistor biased at $(A)$ maximum $\left(K_{V}=0.042\right)$ and at minimum $(B)\left(K_{V}\right.$ $=0$ ) charge sensitivity. Values below $\approx 20 \mathrm{mHz}$ are erroneous due to the resolution bandwidth $\mathrm{BW}=19 \mathrm{mHz}$ of the spectrum analyzer. Curve $(A)$ shows a $1 / f^{1 / 2}$ noise spectrum with clear saturation for $f<100 \mathrm{mHz}$ to a value of $V_{N}=4.5 \mu \mathrm{V} / \mathrm{Hz}^{1 / 2}$ corresponding to $Q_{N} \approx 10 \times 10^{-3} \mathrm{e} / \mathrm{Hz}^{1 / 2}$. The spectrum can be fitted with only 4 TLFs. The transistor voltage noise at $(B)$ is smaller or equal to the noise of the voltage amplifier.

the negative slope of the $V\left(V_{g}\right)$ curve, and then in this bias point measured the voltage noise $V_{N}$ at $10 \mathrm{~Hz}$ with a spectrum analyzer. This was repeated for a number of bias current values. From these data, we calculated $Q_{N}$ and $V /\left(e / C_{\Sigma}\right)$. It is noted that $V_{N}$ scales with $K_{V}$ leaving $Q_{N}$ virtually independent of voltage in the region of the $I-V$ curve where there is a significant $V_{g}$ modulation. In other words, the noise is almost independent of the bias current below and in the region of significant gate voltage modulation range and first increases at considerably larger bias currents. The fact that no noise rise is observed around the Coulomb threshold voltage $V /\left(e / C_{\Sigma}\right)=1$ excludes external photon assisted generation of the charge noise and thus implies that internal charge noise dominates at $10 \mathrm{~Hz}$. This is further testified by the fact that we find the same input charge noise $Q_{N}=5 \times 10^{-4} \mathrm{e} / \mathrm{Hz}^{1 / 2}$ at $10 \mathrm{~Hz}$ (typical minimum value at optimal bias) for the same sample mounted with and without the refined HF shielding. The lower frequency part 


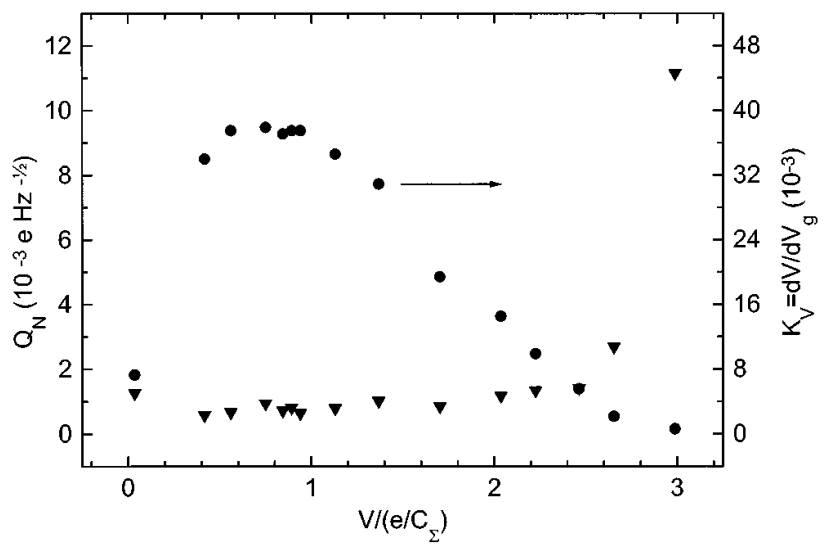

FIG. 2. Experimental charge noise $Q_{N}$ at $10 \mathrm{~Hz}(\boldsymbol{\nabla})$ vs normalized bias voltage. Resolution bandwidth of the spectrum analyzer $\mathrm{BW}=95 \mathrm{mHz} . T$ $=100 \mathrm{mK}$ and $B=0.5 \mathrm{~T}$. Also plotted is the slope $K_{V}=d V / d V_{g}(\bullet)$ of the $V\left(V_{g}\right)$ curve.

of the charge noise spectrum, however, is very sensitive to the effectiveness of the shielding and thus to external disturbances (see below). The charge noise at the relatively high frequency $10 \mathrm{~Hz}$ is qualitatively in agreement with theory ("orthodox theory," no cotunneling). ${ }^{20}$ A quantitative comparison is feasible due to the fact that the theory only includes shot noise from the junction current.

Figure 1(a) shows a typical $V\left(V_{g}\right)$ curve consisting of 500 equidistant points recorded in $9 \mathrm{~min}$ (1.08 s/point) when incrementing $V_{g}$ linearly from -10 to $+10 \mathrm{mV}$. The curve is the last in a sequence of five such $V\left(V_{g}\right)$ curves recorded in 45 min only separated by an interval of $\approx 10 \mathrm{~s}$ needed to reset the setup before the next $V_{g}$ ramp. When put on top of each other the five consecutive curves seemingly only differ by the superimposed noise. The experiment thus is very stable and in fact such $V\left(V_{g}\right)$ curves were reproducible for several hours. Such long term stability is unusual for SET devices and proves that our setup permits us to "freeze" a given background charge configuration below $100 \mathrm{mK}$. The oscillation period $\Delta V_{g}$ is $10.6 \mathrm{mV}$ or $260 \mathrm{~s}$ if we regard the $V_{g}$ axis as a time axis. The corresponding fundamental frequency is $3.9 \mathrm{mHz}$. If we apply FFT to each of the five $V\left(V_{g}\right)$ curves both, the amplitudes and the phases calculated at the fundamental frequency agree within experimental uncertainty. Actually a (direct) FFT of the five $V\left(V_{g}\right)$ curves placed on the common time axis can be used (direct) to find $V_{N}(f)$ and thus $Q_{N}(f)$ at frequencies as low as $0.35 \mathrm{mHz}$. For the conversion to charge noise, we use Eq. (1) with the corresponding (and time averaged) value of $K_{V}\left(V_{g}\right)$. To avoid overload, a few points around the top and bottom of the $V\left(V_{g}\right)$ curves where $K_{V}^{-1} \approx 0$ have to be left out. This method $^{2 i}$ quantitatively gives the same spectrum and saturation of the charge noise as obtained with the spectrum analyzer for frequencies above $\approx 10 \mathrm{mHz}$ using a fixed bias point (see below).

Figure 1(b) shows the corresponding voltage noise spectra $(5 \mathrm{mHz}<f<2 \mathrm{~Hz})$ recorded by the spectrum analyzer with SET transistor biased in the two points $A\left(K_{v}\right.$ $=0.042)$ and $B\left(K_{v} \approx 0\right)$ with maximum and minimum charge sensitivity, respectively. Due to the resolution band- width, $\mathrm{BW}=19 \mathrm{mHz}$, the steep rise of both spectra below $\approx 20 \mathrm{mHz}$ is erroneous and should be disregarded. The spectrum analyzer numerically averages 10 spectra each recorded during $200 \mathrm{~s}$.

The differential amplifier voltage noise spectrum, which is measured with a resistor $(0-100 \mathrm{k} \Omega, 300 \mathrm{~K})$ substituting the SET transistor, closely agrees with its specified typical value. At $1 \mathrm{~Hz}$, the $(B)$ curve is $3-6 \mathrm{~dB}$ higher than the measured amplifier noise, meaning that at $1 \mathrm{~Hz}$ the noise from the SET transistor is smaller or equal to the amplifier noise. The measured amplifier voltage noise spectrum deviates from a $1 / f^{1 / 2}$ dependence in the same way as the $(B)$ curve implying that the voltage noise from the SET transistor also in bias point $(B)$ is not far from a $1 / f^{1 / 2}$ dependence. This SET transistor $1 / f^{1 / 2}$ noise in point $(B)$ cannot be ordinary charge noise since $K_{v}=0$, but it may originate from TLFs or traps directly affecting the tunneling probability. Junction resistance fluctuations and bias current shot noise both have white noise spectra and thus seems less likely. Whether cotunneling $^{16}$ or the internal anticorrelation ${ }^{20}$ between the current and voltage noise spectra can explain this excess noise is presently disputed. ${ }^{22}$

The top spectrum $(A)$ in Fig. 1(b) shows a $1 / f^{1 / 2}$ dependence with a clear saturation at $V_{N} \approx 4.5 \mu \mathrm{V} / \mathrm{Hz}^{1 / 2}$ for $f$ $<100 \mathrm{mHz}$ corresponding to a charge noise $Q_{N} \approx 10$ $\times 10^{-3} \mathrm{e} / \mathrm{Hz}^{1 / 2}$. For the conversion to charge noise is used the measured $K_{v}=0.042, C_{g}=15 \mathrm{aF}$, and amplifier gain 100 (=40 dB). A $Q_{N}(f)$ spectrum of this type can be modelled using a large number of TLFs with equal amplitude (as probably defined by the equilibrium temperature) and randomly distributed relaxation times. ${ }^{3,13}$ Using the standard DebyeLorentzian spectral distribution of a single TLF, one gets

$$
Q_{N}(f)=\left[\Sigma \Delta Q_{i}^{2} \tau_{i} /\left(4 \pi^{2} f^{2} \tau_{i}^{2}+1\right)\right]^{1 / 2},
$$

where $\Delta Q_{i}$ and $\tau_{i}$ is the amplitude and relaxation time of the $i$ th TLF. Using Eq. (2), an addition of only four TLFs with the same amplitude $\Delta Q_{i}=6.1 \times 10^{-3}$ e and relaxation times $\tau_{i}=2 \mathrm{~s}, 0.5 \mathrm{~s}, 125 \mathrm{~ms}$, and $60 \mathrm{~ms}$, respectively, gives a fairly good fit to the $(A)$ spectrum in Fig. 1(b). It is noted that a single TLF gives a $1 / f$ slope for $f \gg 1 / \tau$, and a "knee" frequency $f=1 /(2 \pi \tau)$.

Very few published SET transistor noise spectra show saturation at low frequencies. This may be due to insufficient shielding or an initial high level of active charge traps created during the rapid cooling in top loaded cryostats (see below). Also measurements in the "constant charge" mode, which (in analogy to the "constant flux" mode in SQUIDs) conveniently makes the transistor response linear, may lead to parametrically generated low frequency noise when the noise $V_{N}(t)$ is comparable to the used modulation amplitude, which in turn must be much smaller than the $V\left(V_{g}\right)$ modulation. To our knowledge, saturation from a $1 / f^{1 / 2}$ spectrum has only been reported by two groups in a $\mathrm{Al} / \mathrm{AlO}_{x} / \mathrm{Al} \mathrm{SET}$ transistor. $^{3,23}$ Saturation of this kind, however, seems quite common in semiconductor quantum dots. ${ }^{15}$ The understanding of defects and traps leading to charge noise of the "telegraph" in SET devices is of particular importance for applications within metrology and SET electronics. ${ }^{24,25}$ 
A thermal cycling of the sample to $4.2 \mathrm{~K}$ [as between the measurements depicted in Figs. 1(a) and 3] always leads to a changed voltage noise pattern and often also to a "phase shift" of the $V\left(V_{g}\right)$ curve. The thermal cycling may even establish approximately the same high noise level in the low and high sensitivity regions of the $V\left(V_{g}\right)$ curve. Heating to $300 \mathrm{~K}$ surely gives changes corresponding to dramatic changes in the background charge distribution. Many experiments have reported that the charge noise decreases with time (on the scale of hours or even weeks) after cooling to base temperature. In order to study this relaxation phenomena, we have performed a series of thermal cycles with the same sample. Our nontop-loaded KelvinOx ${ }^{\circledR}$ dilution refrigerator allows us to cool the sample very slowly (usually over two nights) from room temperature to $4.2 \mathrm{~K}$ and further to base temperature in a very controlled way. As described above, the data collection system monitors the temperature of all important parts and especially of the mixing chamber with the attached sample holder. Rapid cooling always leads to high noise levels, while slow cooling usually provides low noise. The cooling rate from 300 to $77 \mathrm{~K}$ seems to be of particular importance. Often we cool the sample holder without exchange gas, meaning that it takes until the next day before it reaches $77 \mathrm{~K}$. Similarly we can cool from 77 to 4.2 $\mathrm{K}$ over many hours.

The reduced noise, indicating a low inherent density of fluctuating background charges, that results from a slow cooling rate may have several explanations. First, the bombardment with photons having slowly decreasing energies, perhaps in combination with the conducting Si substrate and the high aluminium film coverage of the substrate surface, may disarm the dominant TLFs by gradually inactivating ("annealing") the charge traps. Second, the rf shielding provided by the Thermocoax ${ }^{\circledR}$ and the thick-wall copper sample holder, which is effective also at higher temperatures, prevents excitation of charge traps, and so may aid the annealing process. Finally, the slow cooling induces less strain and stress on the sample. This in itself prevents charge trap formation, and mechanically it may even relax dislocations and heal surface defects. The general experimental result of a slow cooling sequence is that one or very few dominant TLFs prevail in the superimposed noise pattern of many low amplitude equilibrium TLFs. The $V\left(V_{g}\right)$ curves in Figs. 1 and 3 show no dominant TLFs.

Figure 3(a) shows an example of a $V\left(V_{g}\right)$ curve with different voltage noise on the positive and negative slope. Since each slope is uniquely associated with one of the junctions, also the enhanced noise on the positive slope must relate to that junction, say $J_{A}$. In order to investigate this further, we have measured the spectra $(5 \mathrm{mHz} \leqslant f \leqslant 2 \mathrm{~Hz}$, $\mathrm{BW}=19 \mathrm{mHz}$ ) shown in Fig. 3(b) with the transistor biased with fixed parameters $\left(I_{B}=+0.5 \mathrm{nA}, T=70 \mathrm{mK}\right)$ at the $V_{g}$ values giving the largest $K_{v}$ on the positive (point $A$ ) and negative (point $B$ ) slopes, respectively. As found above, the junctions have nearly the same capacitance and thus $K_{v}$ has nearly the same value $\left(K_{v}=0.034\right)$ in $(A)$ and $(B)$. Surprisingly, the two voltage noise spectra are identical within experimental uncertainty. The noise at $1 \mathrm{~Hz}$ is $750 \mathrm{nV} / \mathrm{Hz}^{1 / 2}$
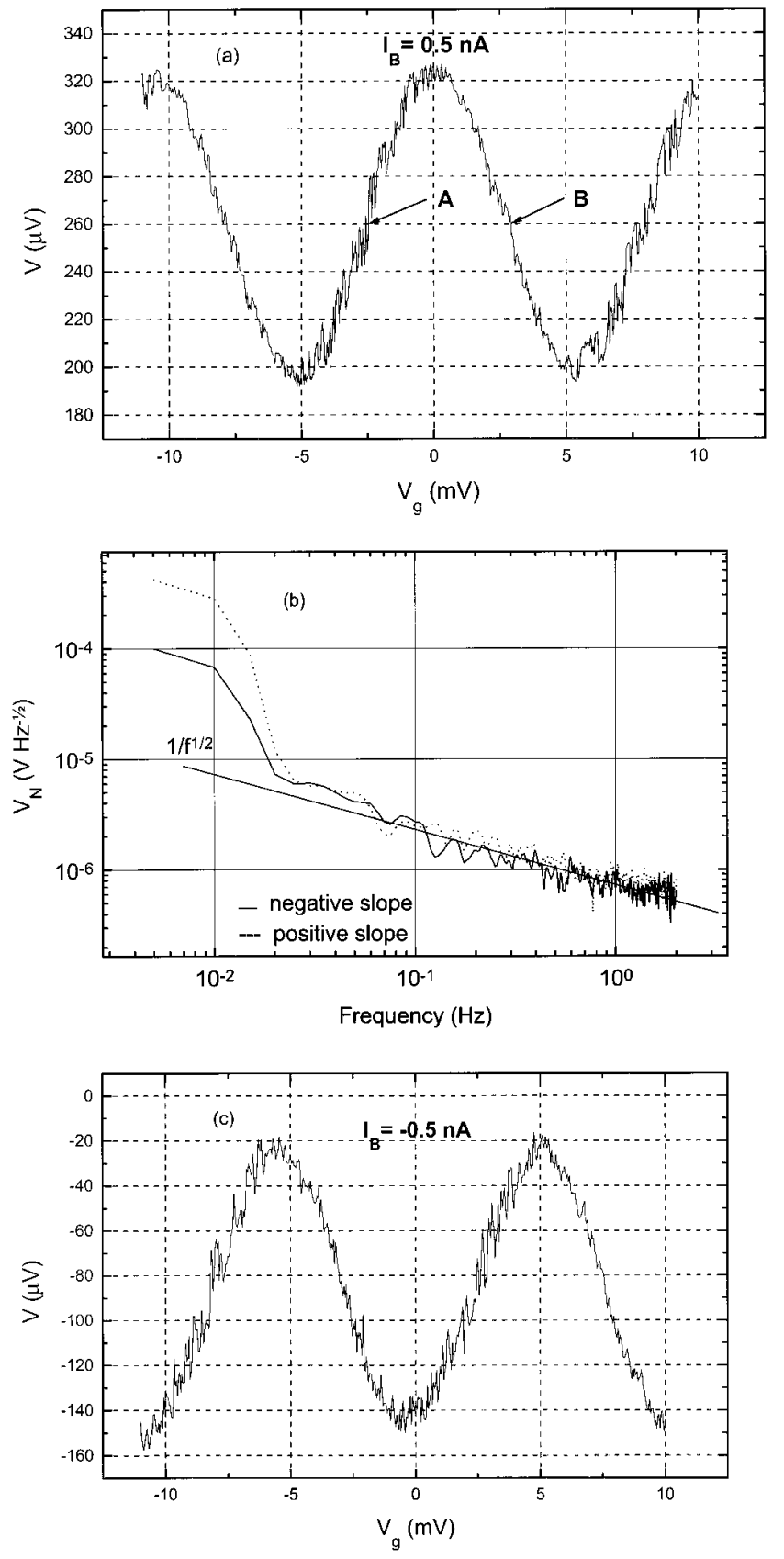

FIG. 3. (a) $V\left(V_{g}\right)$ curve with different noise pattern on the positive and negative slopes $\left(I_{B}=0.5 \mathrm{nA}, T=70 \mathrm{mK}, B=0.5 \mathrm{~T}\right) . V_{g}$ is incremented from -10 to $+10 \mathrm{mV}$. (b) The corresponding voltage noise spectra $(5 \mathrm{mHz} \leqslant f$ $\leqslant 2 \mathrm{~Hz}, \mathrm{BW}=19 \mathrm{mHz}$ ) recorded with the transistor biased at points $(A)$ and $(B)$ are identical within experimental uncertainty. The voltage noise at $1 \mathrm{~Hz}$ is $V_{N}=750 \mathrm{nV} / \mathrm{Hz}^{1 / 2}$ corresponding to $Q_{N}=2.1 \times 10^{-3} \mathrm{e} / \mathrm{Hz}^{1 / 2}$. No saturation is observed at low frequencies. (c) Same as (a) but the $V\left(V_{g}\right)$ curve is recorded with inverted bias current $I_{B}=-0.5 \mathrm{nA}$. As above $V_{g}$ is ramped from -10 to $+10 \mathrm{mV}$. Note that the noise still is largest on the positive slope.

corresponding to $Q_{N}=2.1 \times 10^{-3} \mathrm{e} / \mathrm{Hz}^{1 / 2}$ and no saturation is observed at low frequencies.

Figure 3(c) shows the $V\left(V_{g}\right)$ curve recorded with inverted current bias $\left(I_{B}=-0.5 \mathrm{nA}, T=70 \mathrm{mK}\right)$. The excess noise remains on the positive slope and thus stems from junction $J_{A}$. Spectra (not shown here) recorded with inverted current bias again at maximum $K_{v}$ (here $K_{v}=0.031$ ) were 

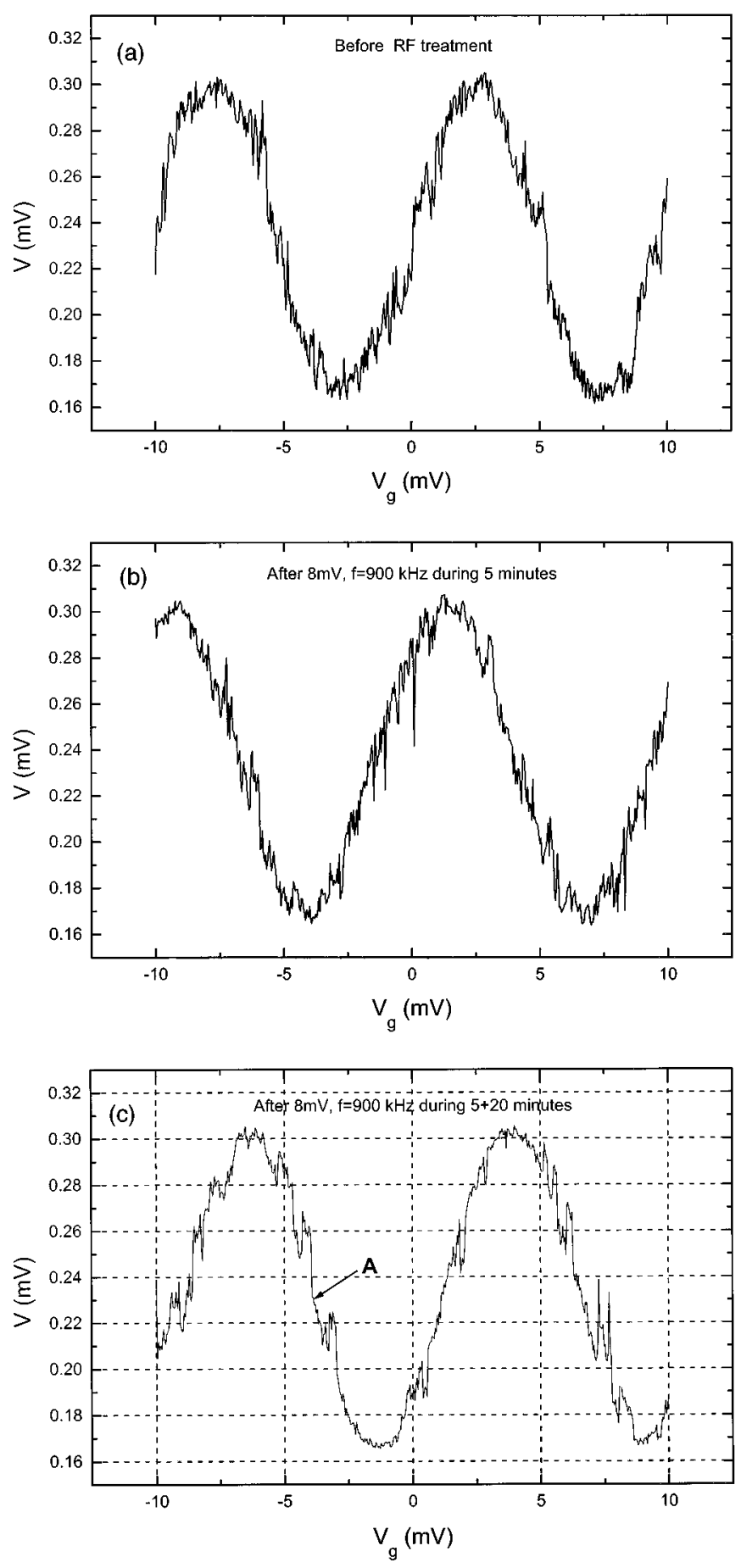

identical to those shown in Fig. 3(b) within experimental uncertainty. The fact that we for the same junction (which for this bias actually is the one, that limits the tunnel current through the SET transistor) observe the same spectral noise for fixed $V_{g}$ on the two slopes but different superimposed voltage noise patterns when ramping $V_{g}$ in our opinion can only be ascribed to a dynamic detrapping of charge in traps very near or inside this junction. The detrapping may be induced by the increasing junction voltage $V$ (increased electrical field) "following" the ramping of $V_{g}$, or alternatively it might be a voltage ( $E$ field) enhanced probability of tunneling mediated by traps inside the junction barrier. Ideally
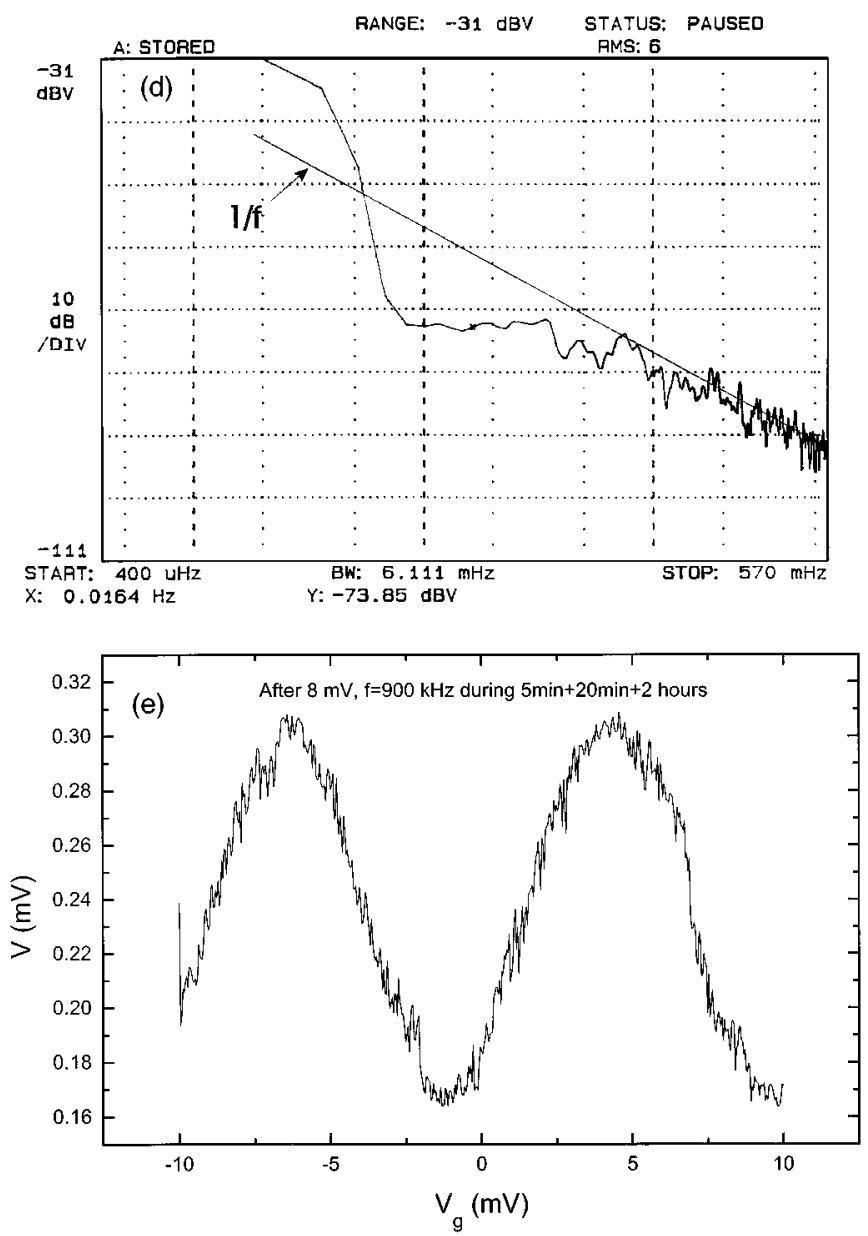

FIG. 4. Sequence of $V\left(V_{g}\right)$ curves for a SET transistor irradiated by 900 $\mathrm{kHz}$ rf supplied to the gate voltage wire. $I_{B}=0.5 \mathrm{nA}, T=70 \mathrm{mK}, B$ $=0.5 \mathrm{~T}$. (a) Before rf treatment. Note the high background noise and the dominant TLF telegraphlike "jumps" at both slopes. (b) After irradiation with rf (amplitude $8 \mathrm{mV}$ measured at room temperature) for $5 \mathrm{~min}$. The dominant jumps have disappeared. (c) After continued rf treatment for another $20 \mathrm{~min}$. A single dominant TLF generates jumps on a less noisy background. (d) Noise spectrum $(400 \mu \mathrm{Hz} \leqslant f \leqslant 570 \mathrm{mHz}, \mathrm{BW}=6.1 \mathrm{mHz})$ recorded with the transistor biased in $(A)$ on the $V\left(V_{g}\right)$ curve [Fig. 4(c)]. There is a clear $1 / f$ dependence. For $f<100 \mathrm{mHz}$, the noise saturates to $V_{N}=25 \mu \mathrm{V} / \mathrm{Hz}^{1 / 2}$ corresponding to $Q_{N}=63 \times 10^{-3} \mathrm{e} / \mathrm{Hz}^{1 / 2}$. The spectrum can be fitted to a single TLF. (e) After continued rf treatment, a noisy background with a different pattern has reappeared. There are no dominant TLFs.

the $V\left(V_{g}\right)$ curve is triangular and it is remarkable that the excess noise on both $V\left(V_{g}\right)$ curves shown in Figs. 3(a) and 4(c) seems to prevail up (down) to the theoretical max (min) points. This is difficult to explain as regular gate charge noise, especially since it does not show the expected $K_{v}$ dependence seen, e.g., in Fig. 1(a) where the noise diminishes as $K_{v}$ approach zero.

The noise is very sensitive to previous rf irradiation. Figure 4 shows successive $V\left(V_{g}\right)$ curves recorded before and after "treatment", with a fixed amplitude $900 \mathrm{kHz}$ rf signal supplied to the gate voltage wire for certain time intervals. The rf amplitude measured at the top flange of the cryostat is 
$8 \mathrm{mV}$. Since the $\mathrm{rf}$ signal is injected along the dc wires, through filters, and the Thermocoax ${ }^{\circledR}$ cables a strong frequency dependence is very likely. We have no means to measure the rf amplitude at the sample but since the impedance between the island and ground is relatively low (of order $50-100 \mathrm{k} \Omega$ ) we believe that the $\operatorname{rf} E$ field is concentrated between the island and the gate electrode. No extensive investigation has been made but the progression depicted in Fig. 4 is typical for the other frequencies and amplitudes we tried. The $V\left(V_{g}\right)$ curves were recorded immediately after the rf signal was switched off.

Figure 4(a) shows a $V\left(V_{g}\right)$ curve with some active TLFs on a rather noisy background, which remains also for $K_{v}$ $=0$. The first rf treatment for 5 min between Figs. 4(a) and 4(b) seemingly "killed" at least one dominant TLF. It is remarkable that the shift of the $V\left(V_{g}\right)$ curve towards more negative $V_{g}$ values apparently regenerates during the recording time ( $9 \mathrm{~min}$ ). Assuming that $C_{1}, C_{2}$, and especially $C_{g}$ are constant a plausible explanation could be that the (many?) other relatively low energy charge traps (excited by the $\mathrm{rf}$ signal) relax to their original states within a few minutes.

The next rf treatment for 20 min [Fig. 4(c)] radically reduces the background charge noise, now being very small near $K_{v}=0$. Now there is one dominant TLF, which is particularly visible in the regions near the maximum slope $\left(K_{V}=0.038\right)$. The modulation depth remains unchanged but compared to Figs. 4(a) and 4(b) there is a permanent shift of the $V\left(V_{g}\right)$ curve towards more positive $V_{g}$ values. Just after recording Fig. 4(c), the noise spectrum was measured with fixed parameters [point $A$ in Fig. 4(c)]. The spectrum analyzer output $(0.4 \mathrm{mHz} \leqslant f \leqslant 570 \mathrm{mHz}, \mathrm{BW}=6.1 \mathrm{mHz})$ shown in Fig. 4(d) has a clear $1 / f$ dependence and saturates for $f$ $\leqslant 100 \mathrm{mHz}$ to a voltage noise $V_{N}=25 \mu \mathrm{V} / \mathrm{Hz}^{1 / 2}$ corresponding to a charge noise $Q_{N}=63 \times 10^{-3} \mathrm{e} / \mathrm{Hz}^{1 / 2}$. This spectrum can be modelled using Eq. (2) by a single TLF with amplitude $\Delta Q_{1}=37 \times 10^{-3}$ e and relaxation time $\tau_{1}=2.8 \mathrm{~s}$.

The $V\left(V_{g}\right)$ curve in Fig. 4(e) obtained after further rf exposure for another $2 \mathrm{~h}$ is identical to the curve in Fig. 4(c) with respect to modulation depth and phase shift. The only changes are that the single dominant TLF has disappeared and that the background noise has increased somewhat (also at $K_{V}=0$ ), actually to a level being only marginally smaller than before the rf treatment. It is likely that there are two types of charge traps causing the noise, one kind is "cured" by the initial rf exposure while extended rf treatment creates the second type. Perhaps, as with hysteretic effects, we should gradually reduce the rf amplitude in order to achieve the desired "annealing" to lower noise.

\section{SUMMARY}

An efficient HF shielding including resistive coaxial lines permits us to preserve and study for many hours a given background charge configuration in a current biased aluminium SET transistors below $\approx 100 \mathrm{mK}$. The samples are fabricated on doped Si substrates with a geometry that impedes formation of surface charge traps. The superimposed voltage noise patterns on the $V\left(V_{g}\right)$ curve and the corresponding voltage noise spectra $(5 \mathrm{mHz}<f<30 \mathrm{~Hz})$ are re- lated to the distribution of the charge traps resulting from a particular cooling sequence or a repetitive rf irradiation. For $f \geqslant 10 \mathrm{~Hz}$, we find that the same input charge noise, typically $Q_{N}=5 \times 10^{-4} \mathrm{e} / \mathrm{Hz}^{1 / 2}$ at $10 \mathrm{~Hz}$, is dominant due to internal charge fluctuations. At lower frequencies, the charge noise depends strongly on the cooling sequence as well as on external disturbances including thermal radiation from the 4.2 $\mathrm{K}$ environment. In our setup, a controlled cooling of the sample with low cooling rate apparently "anneals" the charge traps leading to a particularly low charge noise. Short time rf treatment of the sample after cooling by irradiation with $900 \mathrm{kHz}$ reduces the usual background charge noise while hour-long rf treatments seemingly create another type of noise. Experiments with rf annealing of charge noise are in progress.

Depending on the thermal and rf treatment, we observed charge noise spectra with both $1 / f$ and $1 / f^{1 / 2}$ dependencies. For $f<100 \mathrm{mHz}$, the noise saturates at a $Q_{N}$ value of $(1-5) \times 10^{-2} \mathrm{e} / \mathrm{Hz}^{1 / 2}$. Each spectrum can be fitted using a single or a sum of same-amplitude two-level fluctuators (TLF) having a Debye-Lorentzian spectrum with relaxation times of order seconds.

The found asymmetric noise patterns superimposed on the two slopes of the $V\left(V_{g}\right)$ curve are ascribed to dynamic charge trapping near or inside one of the junctions induced when ramping the gate voltage and thus the junction voltage. Dynamic trapping may limit the high frequency applications of the SET transistor. Also the temperature dependence of $Q_{N}$ is under continued investigation. Preliminary measurements show that $Q_{N}$ is virtually temperature independent for $T<300 \mathrm{mK}$.

\section{ACKNOWLEDGMENTS}

We thank O. Gorbonosov for fabricating the samples at the Laboratory of Cryoelectronics, Moscow State University, Russia, and G. M. Fischer, M. W. Johnson, and V. Kornev for early assistance with the setup. We also thank H. D. Jensen, K. K. Likharev, A. N. Korotkov, L. S. Kuzmin, J. Niemeyer, F. C. Wellstood, and A. B. Zorin for useful comments and discussions. The work has been supported in parts by the Danish Research Academy, Brds. Hartmann Foundation, and the Danish Natural Science Research Council.

${ }^{1}$ D. V. Averin and K. K. Likharev, in Mesoscopic Phenomena in Solids, edited by B. L. Al'tshuler, P. A. Lee, and R. A. Webb (Elsevier, Amsterdam, 1991).

${ }^{2}$ Single Charge Tunneling, edited by H. Grabert and M. H. Devoret, NATO ASI Series B, Physics (Plenum, New York, 1992), Vol. 294.

${ }^{3}$ S. M. Verbrugh, thesis, DIMES, Delft University of Technology, 1995.

${ }^{4}$ G. Zimmerli, R. L. Kautz, and J. M. Martinis, Appl. Phys. Lett. 61, 2616 (1992).

${ }^{5}$ P. D. Dresselhaus, L. Ji, Siyuan Han, J. E. Lukens, and K. K. Likharev, Phys. Rev. Lett. 72, 3226 (1994).

${ }^{6}$ D. V. Averin and Yu. V. Nazarov, Phys. Rev. Lett. 65, 2446 (1990); T. M. Eiles, G. Zimmerli, H. D. Jensen, and J. M. Martinis, ibid. 69, 148 (1992).

${ }^{7}$ L. S. Kuzmin, Yu. A. Pashkin, A. N. Tavkhelidze, F.-J. Ahlers, T. Weimann, D. Quenter, and J. Niemeyer, Appl. Phys. Lett. 68, 2902 (1996).

${ }^{8}$ E. H. Visscher, S. M. Verbrugh, J. Lindeman, P. Hadley, and J. E. Mooij, Appl. Phys. Lett. 66, 305 (1995).

${ }^{9}$ C. Schönenberger, H. van Houten, and C. W. J. Beenakker, Physica B 189, 218 (1993). 
${ }^{10}$ K. Matsumoto, M. Ishii, K. Segawa, and Y. Oka, Appl. Phys. Lett. 68, 34 (1996).

${ }^{11}$ M. J. Buckingham, in Noise in Electronic Devices and Systems (Wiley, New York, 1983), Chaps. 5 and 6.

${ }^{12}$ A. B. Zorin, F.-J. Ahlers, J. Niemeyer, T. Weimann, H. Wolf, V. A. Krupenin, and S. V. Lotkhov, Phys. Rev. B 53, 13682 (1996).

${ }^{13}$ A. J. Manninen and J. P. Pekola, Czech. J. Phys. 46, 2293 (1996).

${ }^{14}$ G. Zimmerli, R. L. Kautz, and J. M. Martinis, Appl. Phys. Lett. 61, 237 (1992).

${ }^{15}$ C. Dekker, A. J. Scholten, F. Liefrink, R. Eppenga, H. van Houten, and C. T. Foxon, Phys. Rev. Lett. 66, 2148 (1991).

${ }^{16}$ J. M. Martinis, M. Nahum, and H. D. Jensen, Phys. Rev. Lett. 72, 904 (1994), and J. M. Martinis and M. Nahum, Phys. Rev. B 48, 18316 (1994).
${ }^{17}$ P. Wahlgren, P. Delsing, and D. B. Haviland, Phys. Rev. B 52, 2293 (1995).

${ }^{18}$ K. K. Likharev, IEEE Trans. Magn. 23, 1142 (1987).

${ }^{19}$ A. B. Zorin, Rev. Sci. Instrum. 66, 4296 (1995).

${ }^{20}$ A. N. Korotkov, D. V. Averin, K. K. Likharev, and S. A. Vasenko, Springer Series Electron. Phys. 1992.

${ }^{21}$ A. N. Tavkhelidze, J. Mygind, L. S. Kuzmin, and O. Gorbonosov, Czech. J. Phys. 46, 2287 (1996).

${ }^{22}$ A. N. Korotkov (private communication).

${ }^{23}$ N. Zimmerman, NIST, Gaithersburg, (private communication) PTB 1996

${ }^{24}$ G. Zimmerli, R. L. Kautz, and J. M. Martinis, Appl. Phys. Lett. 61, 237 (1992).

${ }^{25}$ D. Song, A. Amar, C. J. Lobb, and F. C. Wellstood, IEEE Trans. Appl. Supercond. 5, 3085 (1995). 